\title{
The essential mycobacterial genes, $f a b G 1$ and $f a b G 4$, encode 3-oxoacyl-thioester reductases that are functional in yeast mitochondrial fatty acid synthase type 2
}

\author{
Aner Gurvitz
}

Received: 26 February 2009 / Accepted: 22 July 2009 / Published online: 14 August 2009

(C) The Author(s) 2009. This article is published with open access at Springerlink.com

\begin{abstract}
Mycobacterium tuberculosis represents a severe threat to human health worldwide. Therefore, it is important to expand our knowledge of vital mycobacterial processes, such as that effected by fatty acid synthase type 2 (FASII), as well as to uncover novel ones. Mycobacterial FASII undertakes mycolic acid biosynthesis, which relies on a set of essential enzymes, including 3-oxoacyl-AcpM reductase FabG1/Rv1483. However, the M. tuberculosis genome encodes four additional FabG homologs, designated FabG2-FabG5, whose functions have hitherto not been characterized in detail. Of the four candidates, FabG4/

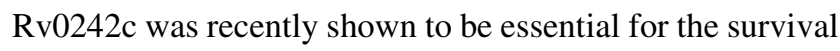
of $M$. bovis BCG. The present work was initiated by assessing the suitability of yeast $\operatorname{oar} 1 \Delta$ mutant cells lacking mitochondrial 3-oxoacyl-ACP reductase activity to act as a surrogate system for expressing FabG1/MabA directed to the mitochondria. Mutant yeast cells producing this targeted FabG1 variant were essentially wild type for all of the chronicled phenotype characteristics, including respiratory growth on glycerol medium, cytochrome assembly and lipoid acid production. This indicated that within the framework of de novo fatty acid biosynthesis in yeast mitochondria, FabG1 was able to act on shorter $\left(\mathrm{C}_{4}\right)$ acyl substrates than was previously proposed $\left(\mathrm{C}_{8-20}\right)$ during mycolic acid biosynthesis in M. tuberculosis. Thereafter, FabG2-FabG5 were expressed as mitochondrial proteins in the oarl $\Delta$ strain, and FabG4 was found to complement the mutant
\end{abstract}

Communicated by G. Klug.

A. Gurvitz $(\square)$

Section of Physiology of Lipid Metabolism, Institute of Physiology, Center for Physiology,

Pathophysiology and Immunology, Medical University of Vienna,

Schwarzspanierstrasse 17, 1090 Vienna, Austria

e-mail: aner.gurvitz@meduniwien.ac.at phenotype and contain high levels of 3-oxoacyl-thioester reductase activity. Hence, like FabG1, FabG4 is also an essential, physiologically functional 3-oxoacyl-thioester reductase, albeit the latter's involvement in mycobacterial FASII remains to be explored.

Keywords Mycobacterium tuberculosis. Rv0242c-Rv1350-Rv1483-Rv2002-Rv2766c . MabA · FASII-FAS II-FAS-II · Lipoic acid
Abbreviations
ACP Acyl carrier protein
AcpM Mycobacterial ACP
BCG Bacillus Calmette-Guérin
FAS Fatty acid synthase
FASI Type 1 associative FAS
FASII Type 2 dissociative FAS
MLS Mitochondrial leader sequence
ORF Open reading frame
PCR Polymerase chain reaction
MFE2 Peroxisomal type 2 multifunctional enzyme

\section{Introduction}

Mycobacterium tuberculosis is the main cause of death due to a single infectious agent (World Health Organisation 1997) among humans, and as many as 2 billion people worldwide are infected with tuberculosis (for a review, see Takayama et al. 2005 and references cited therein). In light of the rise in mycobacterial resistance toward isoniazid, which represents one of less than a handful of first-line antituberculous drugs, there is an urgent need to widen the study of vital M. tuberculosis processes so as to develop novel drugs. 
Isoniazid targets the production of mycolic acids (Banerjee et al. 1994), which are very long-chain $\alpha$-branched $\beta$-hydroxylated fatty acids $\left(\mathrm{C}_{54-63}\right)$ that help form the protective layer around this pathogen, thereby adding to its persistence and virulence (Takayama et al. 2005). Mycolic acid biosynthesis involves a combination of an associative type 1 fatty acid synthase (FASI) similar to the system found in the eukaryotic cytosol, in that it comprises several enzymatic activities located on a single multifunctional synthase (Bloch 1977) and a dissociative type 2 process (FASII) resembling the plant-plastid system that consists of separate enzymes. FASII also occurs in yeast mitochondria (reviewed in Hiltunen et al. 2005).

In M. tuberculosis FASII, FabG1 (MabA) undertakes the reduction of 3-oxoacyl-acyl carrier protein (AcpM) to generate 3-hydroxyacyl-AcpM (Banerjee et al. 1998), whereas in the equivalent mitochondrial process in Saccharomyces cerevisiae, this step is catalyzed by Oarlp (Schneider et al. 1997). In reference to substrate specificities, FabG1 has been demonstrated previously to catalyze the NADP $(\mathrm{H})$ specific reduction of long-chain $\left(\mathrm{C}_{8-20}\right)$ 3-oxoacyl-thioester species. Molecular modeling of FabG1 revealed a large substrate-binding pocket capable of accommodating longchain substrates (Marrakchi et al. 2002), while yeast Oar1p is presumed to act on only short-chain ones. Analysis of the complete sequence of the M. tuberculosis genome (Cole et al. 1998) revealed FabG1 as having four homologs, termed FabG2-FabG5 (Fig. 1), but, so far, not one of them has been fully characterized.

The essential requirement for FabG activity has been addressed before in a genome-wide survey using transposon-site hybridization, which revealed just over 600 M. tuberculosis genes that were required for optimal growth (Sassetti et al. 2003), among them fabG2/Rv1350. However, a subsequent study identifying almost $200 \mathrm{M}$. tuberculosis genes that were required for mycobacterial survival during infection did not pick up a single $f a b G$ locus (Sassetti and Rubin 2003). The dispute surrounding the requirement for 3-oxoacyl-thioester activity in mycobacteria was finally settled with the finding that FabG1 is in fact essential for M. tuberculosis survival (Parish et al. 2007), whereas in M. bovis BCG, FabG1 and the hitherto uncharacterized FabG4 have both been exposed as being vital (Beste et al. 2009).

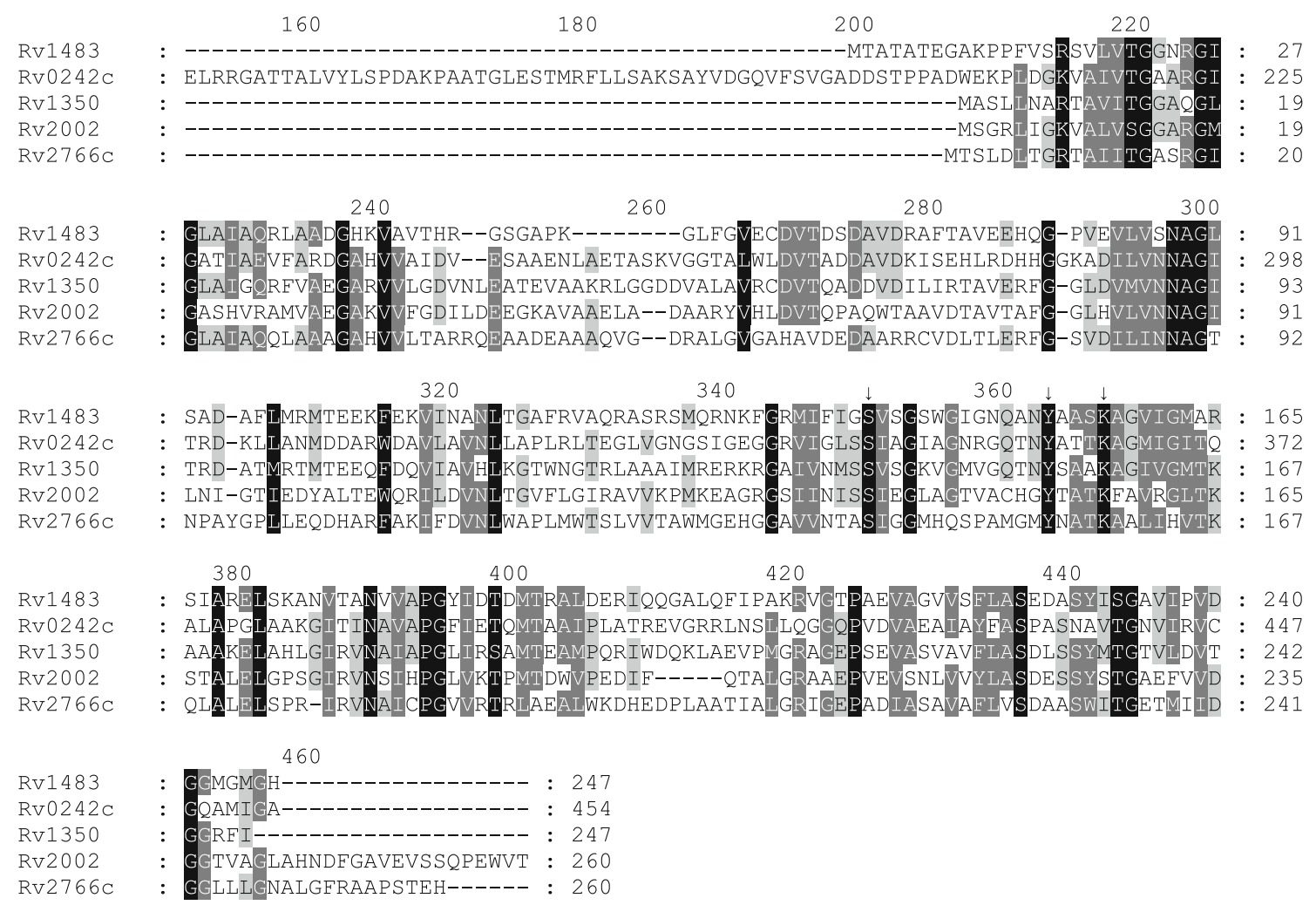

Fig. 1 Homology study of M. tuberculosis FabG proteins. Multalinand Genedoc-based comparison of the deduced amino acid sequence of FabG1 (Rv1483) with those of its homologs FabG2 (Rv1350), FabG3 (Rv2002), FabG4 (Rv0242c) and FabG5 (Rv2766c). Dashes indicate the arrangement of the sequences for best fit, and arrows point to the amino acids forming the catalytic triad (Marrakchi et al. 2002).
The first 150 amino acid residues of FabG4 do not match any of those of the other homologs, and were removed from the figure. Black shadings refer to strictly conserved amino acid residues among all the sequences, whereas the darker and lighter gray shadings denote regions with more relaxed residue similarities not necessarily shared by the full set of sequences 
The latter finding of two non-redundant FabGs in $M$. bovis BCG raised the pertinent question of whether a similar situation might also occur in M. tuberculosis, whereby FabG1 is not necessarily the sole essential 3-oxoacyl-thioester reductase after all. This prompted the deployment of an alternative strategy for studying the functions of the remaining four FabG homologs. To this end, S. cerevisiae was exploited as a surrogate host for investigating $M$. tuberculosis proteins (Gerum et al. 2002) with potential FASII activities. Here, mitochondrially targeted versions of FabG1 to FabG5 were expressed in yeast oarld mutant cells (Schneider et al. 1997), which lack 3-oxoacyl-ACP reductase activity and do not respire or grow on glycerol, assemble cytochrome complexes or produce lipoic acid. The effects were compared to those of ectopically expressing native Oar1p. This oarl $\Delta$ strain was applied previously to expose the identity of nematode and human 3-oxoacylthioesters (Gurvitz 2009; Chen et al. 2009). The outcome of this study is discussed in terms of the identification of the essential mycobacterial $f a b G 4$ gene as encoding a novel 3-oxoacyl-thioester reductase, as well as the role of protein-protein interactions in mycobacterial FASII.

\section{Materials and methods}

S. cerevisiae strains, plasmids and oligonucleotides

Yeast strains, plasmids and oligonucleotides used are listed in Table 1. The Escherichia coli strain TOP10 $\mathrm{F}^{\prime}$ was used for all plasmid amplifications and isolations. The wild-type yeast strain BY4741 and its oarl $\Delta$ derivative were obtained from EUROSCARF (www.uni-frankfurt.de). To generate strains yPLM37 to yPLM43, plasmids were introduced into BY4741oarl $\Delta$ cells using a published protocol (Chen et al. 1992), and transformants were selected on solid synthetic defined glucose (SD-Ura) medium lacking uracil.

\section{Plasmid constructions}

DNA manipulations and plasmid constructions were performed according to standard techniques (Ausubel et al. 1989). The five $f a b G$ genes were isolated from H37Rv genomic DNA by polymerase chain reaction (PCR) using Phusion high-fidelity DNA polymerase (Finnzymes Oy, Espoo, Finland) and the corresponding oligonucleotides (Table 1), as described previously (Gurvitz et al. 2008a,b, 2009). Electrophoretic resolution of the PCR products, and their excision, purification and subsequent ligation to an EcoRV-digested pBluescript plasmid vector (Stratagene, La Jolla, CA) were performed as done previously (Gurvitz et al. 2008b). The five pBluescript inserts were subcloned following digestion with NcoI and HindIII restriction enzymes, and ligated behind the CTAl promoter within a similarly digested plasmid pYE352:mitQOR (pPLM62). As a result of this digestion, the $Q O R$ open reading frame (ORF) encoding $E$. coli quinone reductase was removed to leave behind the nucleotides for the yeast Coq3p (Hsu et al. 1996) mitochondrial leader sequence (MLS), as described in the literature (Torkko et al. 2001; Gurvitz et al. 2008b). Following import into the mitochondria, the fused MLS is cleaved off to leave behind a shorter fusion protein preceded by eight amino acids remaining from the MLS fusion (Torkko et al. 2001). Nucleotide sequencing of the fabG inserts verified that no mutations were introduced during the amplification process and that the COQ3-fabG junction remained intact. Construction of plasmid YEp352:OAR1 (pPLM188) expressing S. cerevisiae mitochondrial OARI from the CTAl promoter is described (Chen et al. 2009). Briefly, the $O A R 1$ ORF was amplified from yeast genomic DNA using standard procedure and, following digestion with the appropriate restriction enzymes, the PCR product was ligated to a similarly digested pYE352:CTA1 (Filppula et al. 1995), from which the CTAl ORF was removed (Chen et al. 2009).

Media and growth conditions

Standard yeast (Rose et al. 1990) and E. coli (Sambrook et al. 1989) media were prepared as described. S. cerevisiae strains were propagated on solid, rich glucose medium YPD consisting of $1 \%$ (wt/vol) yeast extract, $2 \%$ (wt/vol) peptone (YP), 2\% (wt/vol) D-glucose and 2\% (wt/vol) agar. YEp352-based episomal plasmids marked with the URA3 gene (Hill et al. 1986) were maintained in transformed strains on solid, synthetic defined (SD-Ura) medium consisting of $0.67 \%$ (wt/vol) yeast nitrogen base without amino acids, $2 \%$ (wt/vol) D-glucose and 3\% (wt/vol) agar, with all necessary supplements added except for uracil (SigmaAldrich Inc., MO, USA). Synthetic complete glycerol (SCglycerol) medium was prepared essentially as SD-Ura, except that uracil was added and glucose was replaced with $3 \%$ (wt/vol) glycerol. Induction of yeast cells in oleic acid medium has been described previously (Gurvitz et al. 1997).

\section{Miscellaneous}

For 3-oxoacyl-thioester reductase activity assays, soluble protein extracts were prepared from yeast cells that were propagated in oleic acid medium and processed as described (Gurvitz et al. 2008a,b, 2009). Protein concentrations were determined according to a published method (Bradford 1976). Reductase activity was assayed spectrophotometrically at $23^{\circ} \mathrm{C}$ as described (Dommes and Kunau 1984). The reductase activity assay mixture consisted of 
Table 1 S. cerevisiae strains, plasmids and oligonucleotides used

\begin{tabular}{|c|c|c|}
\hline Strain, plasmid, oligonucleotide & Description & Source or reference \\
\hline \multicolumn{3}{|l|}{ S. cerevisiae } \\
\hline (1) BY4741 & MATa his $3 \Delta 1$ leu $2 \Delta 0$ met $15 \Delta 0$ ura $3 \Delta 0$ & EUROSCARF \\
\hline (2) BY4741oarl $\Delta^{1 \mathrm{a}}$ & ykl055c::kanMX & EUROSCARF \\
\hline $\mathrm{yPLM} 37^{2}$ & Expressing peroxisomal catalase A (Cta1p) from pPLM187 & This study \\
\hline yPLM38 ${ }^{2}$ & Expressing mitochondrial Coq3p-FabG1 from pPLM189 & This study \\
\hline yPLM39² & Expressing mitochondrial Coq3p-FabG2 from pPLM190 & This study \\
\hline yPLM $40^{2}$ & Expressing mitochondrial Coq3p-FabG3 from pPLM191 & This study \\
\hline yPLM $41^{2}$ & Expressing mitochondrial Coq3p-FabG4 from pPLM192 & This study \\
\hline yPLM $42^{2}$ & Expressing mitochondrial Coq3p-FabG5 from pPLM193 & This study \\
\hline yPLM43 $3^{2}$ & Expressing mitochondrial Oar1p from pPLM188 & This study \\
\hline \multicolumn{3}{|l|}{ Plasmid } \\
\hline (3) pBluescript KS III & pKS cloning vector & Stratagene \\
\hline pPLM83 33 & pKS:Rv1483 mitochondrial $f a b G 1$ in pBluescript & This study \\
\hline pPLM82 3 & pKS:Rv1350 mitochondrial $f a b G 2$ in pBluescript & This study \\
\hline pPLM86 ${ }^{3}$ & pKS:Rv2002 mitochondrial $f a b G 3$ in pBluescript & This study \\
\hline pPLM78 ${ }^{3}$ & pKS:Rv0242c mitochondrial fabG4 in pBluescript & This study \\
\hline pPLM96 ${ }^{3}$ & pKS:Rv2766c mitochondrial $f a b G 5$ in pBluescript & This study \\
\hline (4) YEp352 & URA3-marked multicopy plasmid & Hill et al. (1986) \\
\hline (5) $\mathrm{pPLM} 187^{4}$ & CTA1 behind its promoter (pYE352:CTA1) & Filppula et al. (1995) \\
\hline pPLM188 & $O A R 1$ behind the $C T A 1$ promoter (pYE352:OAR1) & Chen et al. (2009) \\
\hline (6) pPLM625 & $C O Q 3-Q O R$ fusion behind the $C T A 1$ promoter & Torkko et al. (2001) \\
\hline pPLM189 & $C O Q 3-f a b G 1$ fusion behind the $C T A 1$ promoter & This study \\
\hline pPLM190 6 & $C O Q 3-f a b G 2$ fusion behind the $C T A 1$ promoter & This study \\
\hline pPLM191 ${ }^{6}$ & $C O Q 3-f a b G 3$ fusion behind the $C T A 1$ promoter & This study \\
\hline pPLM192 ${ }^{6}$ & $C O Q 3-f a b G 4$ fusion behind the $C T A 1$ promoter & This study \\
\hline pPLM193 ${ }^{6}$ & $C O Q 3-f a b G 5$ fusion behind the $C T A 1$ promoter & This study \\
\hline \multicolumn{3}{|l|}{ Oligonucleotide } \\
\hline Rv1483 MLS-FabG1 F & 5'-TTATCCATGGCTGCCACAGCCACTGAAGGGGC-3' & This study \\
\hline Rv1483 FabG1 R & 5'-TATTAAGCTTCAGTGGCCCATACCCATGCC-3' & This study \\
\hline Rv1350 MLS-FabG2 F & 5'-TTATCCATGGCGTCATTGCTGAACGCTC-3' & This study \\
\hline Rv1350 FabG2 R & 5'-TATACTCGAGTCATATGAACCGGCCGCCAG-3' & This study \\
\hline Rv2002 MLS-FabG3 F & 5'-TTATCCATGGCTGGACGGTTGATAGGAAAGG-3' & This study \\
\hline Rv2002 FabG3 R & 5'-TATTAAGCTTACGTCACCCATTCCGGCTGCG-3' & This study \\
\hline Rv0242c MLS-FabG4 F & 5'-TTATCCATGGCTCCCAAGCGTTCGTCCGATC-3' & This study \\
\hline Rv0242c FabG4 R & 5'-TATTAAGCTTCACGCGCCGATCATGGCCTGGC-3' & This study \\
\hline Rv2766c MLS-FabG5 F & 5'-TTATTATCATGACCTCACTGGATCTGACC-3' & This study \\
\hline Rv2766c FabG5 R & 5'-TTATTATCATGACCTCACTGGATCTGACC-3' & This study \\
\hline
\end{tabular}

${ }^{a}$ The numbers in superscript following the designation of the strains refer to their parental genotypes, e.g., BY4741oarl $\Delta^{1}$ was derived from (1) BY4741. The same principle applies to plasmids

$50 \mathrm{mM} \mathrm{KP} \mathrm{i}_{\mathrm{i}}(\mathrm{pH} 8.0)$ and $50 \mu \mathrm{g} / \mathrm{ml}$ bovine serum albumin, $2.0 \mu \mathrm{l}$ purified protein representing the hydratase 2 domain from Drosophila melanogaster MFE2 (unpublished), $125 \mu \mathrm{M} \mathrm{NADP}{ }^{+}$or $\mathrm{NAD}^{+}$, and $60 \mu \mathrm{M}$ 2-trans-decenoyl$\mathrm{CoA}$ or 2-trans-hexenoyl-CoA that were synthesized via the mixed anhydride system (Goldman and Vagelos 1961). Reduction of the electron carrier was monitored spectrophotometrically at $340-385 \mathrm{~nm}$. The assembly of cytochrome complexes was followed with a spectrophotometer at 480-640 nm, using yeast cells that were applied as a thick paste onto the glass surface of an otherwise aluminum cold-temperature cuvette, which was chilled in liquid nitrogen (Lindenmayer and Estabrook 1958). The lipoic acid content of yeast strains was monitored by a biological assay as described previously (Brody et al. 1997; Hayden et al. 1993). Operon prediction was undertaken using 
OperonDB (http://operondb.cbcb.umd.edu/cgi-bin/operondb/ operons.cgi). Multalin (npsa-pbil.ibcp.fr/cgi-bin/npsa_automat. pl?page =/NPSA/npsa_multalin.html) and Genedoc (www. nrbsc.org/gfx/genedoc/index.html) were used to generate the sequence comparison in Fig. 1.

\section{Results}

Mycobacterial FabG1 and FabG4 restore respiratory growth of $S$. cerevisiae oarl $\Delta$ cells

The M. tuberculosis genome (Cole et al. 1998) contains five genes with the potential to encode 3-oxoacyl-AcpM reductases, termed fabGl to fabG5 (Takayama et al. 2005). FabG1 is chronicled as a 3-oxoacyl-AcpM reductase involved in mycolic acid biosynthesis (Banerjee et al. 1998; Marrakchi et al. 2002); however, the function of the remaining four homologs has hitherto remained unknown. To determine whether these could encode 3-oxoacyl-thioester reductases, FabG1-FabG5 were expressed as fusion constructs that were preceded by the Coq3p mitochondrial leader sequence (Hsu et al. 1996) so as to generate mitochondrially targeted versions. The five fusions were tethered behind the promoter of the CTAl gene encoding peroxisomal catalase A (Ctalp), which is only moderately de-repressed on non-fermentable carbon sources such the glycerol is used here for complementation assays (Filipits et al. 1993; Rottensteiner et al. 1996). These tests were performed on yeast cells lacking Oar1p that have been exploited previously to demonstrate the physiological function of ectopically expressed 3-oxoacyl-ACP reductases (Gurvitz 2009; Chen et al. 2009).

Mutant oarl $\Delta$ yeast cells were transformed with multicopy episomal plasmids loaded with the fusion constructs. Following selection on synthetic defined glucose medium lacking uracil (SD-Ura), survivors were streaked on synthetic complete medium containing glycerol (SCglycerol) as the sole carbon source. Only those mutants expressing FabG1 or FabG4 were capable of forming single colonies on this medium type. A dedicated analysis of the reasons for the failure of FabG2, FabG3 or FabG5 to achieve phenotype rescue was beyond the scope of the present study, these causes being all the more enigmatic since no obvious differences among the homologs emerged from the data shown in Fig. 1.

To demonstrate more accurately that expression of mitochondrial versions of FabG1 or FabG4 in the oarl $\Delta$ mutant cells could compensate for the missing activity attributed to native Oar1p, the four strains (each expressing FabG1, FabG4, Oar1p or Cta1p) were propagated overnight in liquid SD-Ura medium. Following tenfold serial dilution, cultures were spotted onto solid SD-Ura (glucose) or
SCglycerol (glycerol) media, and the plates were incubated at $30^{\circ} \mathrm{C}$ until single colonies were detectable (Fig. 2). As anticipated, mutant oarls cells expressing Oar1p could form single colonies on glycerol, whereas those cells expressing Ctalp were not able to proliferate on this medium, vindicating the experimental design. Importantly, the results demonstrated that the respiratory deficient phenotype of the oar $1 \Delta$ mutant could be rescued by FabG1 and FabG4, indicating functional complementation.

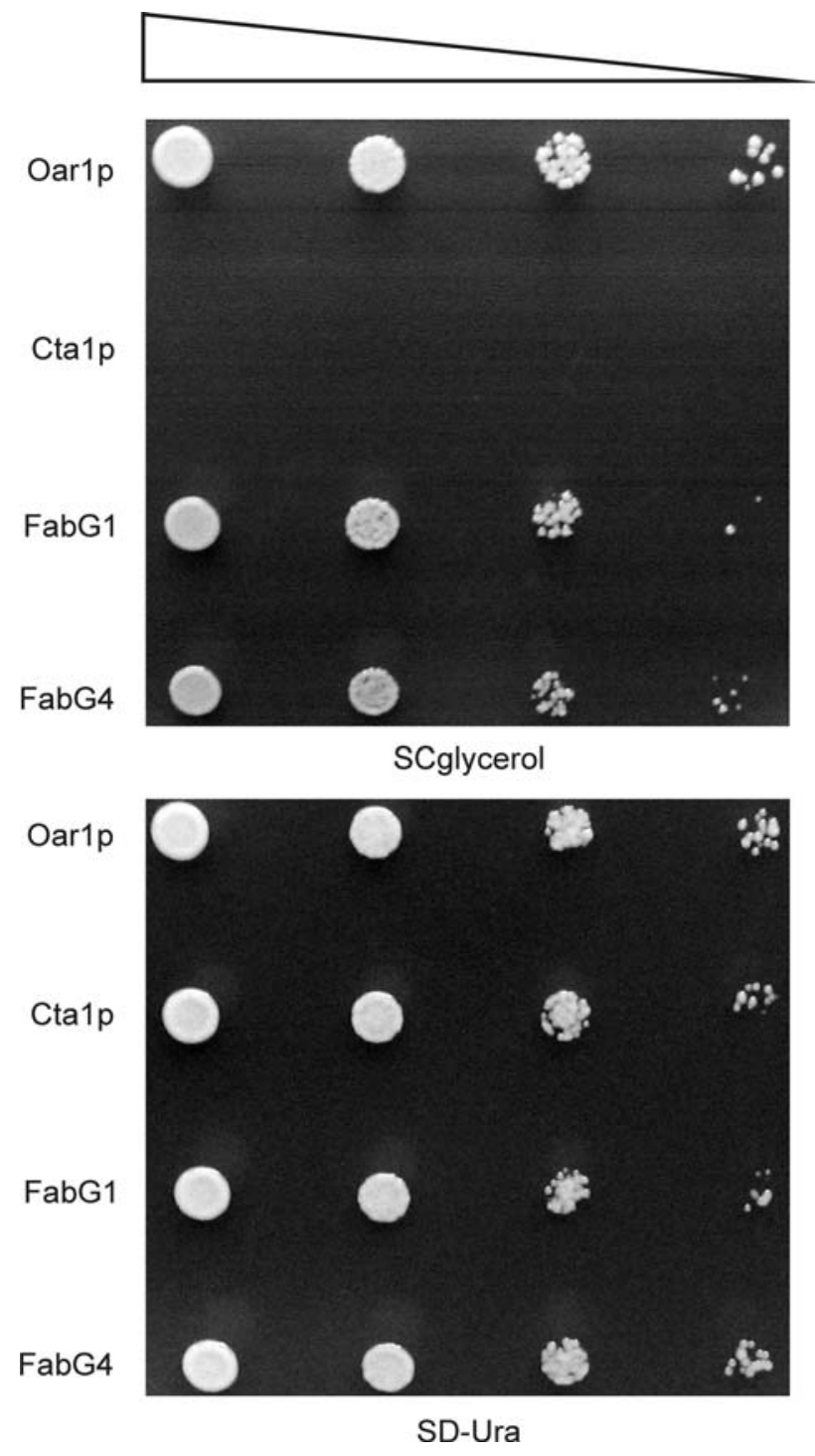

Fig. 2 Growth of S. cerevisiae oar $1 \Delta$ mutants expressing M. tuberculosis FabG1 or FabG4. Yeast oarl $\Delta$ cells expressing native mitochondrial 3-oxoacyl-ACP reductase (Oarlp positive control), native peroxisomal catalase A (Ctalp negative control), mitochondrially targeted FabG1 or mitochondrially targeted FabG4 were grown in liquid SD-Ura medium that selected for plasmid presence. Following serial dilution, cells were applied to solid (SD-Ura) glucose or (SCglycerol) glycerol media. The plates were incubated at $30^{\circ} \mathrm{C}$ until single colonies appeared and recorded photographically. The yPLM strains used were $37,38,41$ and 43 
Yeast oar $1 \Delta$ cells expressing FabG4 contain

3-oxoacyl-thioester reductase activity

Soluble protein extracts from BY4741oarl $\Delta$ cells expressing M. tuberculosis FabG1 or FabG4 were examined for 3-oxoacyl-thioester reductase activity using 2-transhexenoyl-CoA (trans- $\mathrm{C}_{6: 1(2)}$ ) or 2-trans-decenoyl-CoA (trans- $\mathrm{C}_{10: 1(2)}$ ) as substrates. The reactions additionally included purified protein consisting of the hydratase 2 domain from Drosophila melanogaster peroxisomal type 2 multifunctional enzyme (MFE2), which hydrated the substrates to generate the 3-hydroxyacyl-CoA species that could be metabolized, in the reverse reaction, by the 3-oxoacyl-thioester reductases examined. The reactions were monitored spectrophotometrically at wavelengths of 340$385 \mathrm{~nm}$, by following the reduction of $\mathrm{NADP}^{+}$or $\mathrm{NAD}^{+}$to their corresponding protonated species (Gurvitz 2009). Both electron carrier types were considered in this assay, as recent work revealed that 3-oxoacyl-thioester reductase activity might actually depend on $\mathrm{NAD}(\mathrm{H})$, poignantly evidenced by the nematode and human 3-oxoacyl-ACP reductases of mitochondrial FASII (Gurvitz 2009; Chen et al. 2009).

To obtain high levels of protein expression from the fatty acid-inducible CTA1 promoter (Filipits et al. 1993; Rottensteiner et al. 1996), cells were grown overnight on oleic acid medium. The results of the enzyme assays with 2-trans-hexenoyl-CoA as substrate and $\mathrm{NADP}^{+}$as cofactor (in the presence of hydratase 2 activity) performed on oarl $\Delta$ cells over-expressing native Oarlp or Ctalp indicated that any reductase activity present was below the detection limit of the assay used. Recent work on Oar1p by others revealed that its 3-oxoacyl-thioester reductase activity could only be detected in assays performed on a recombinant protein (Chen et al. 2009). Similarly, it was impossible to detect any activity in soluble protein extracts obtained from oarls cells over-expressing FabG1 when $\operatorname{NADP}(\mathrm{H})$ was supplied as cofactor in the reaction, but this was anticipated since previous measurements were also performed solely on a recombinant protein (Marrakchi et al. 2002). Surprisingly, a volume of $0.1 \mu \mathrm{l}$ of soluble protein extract containing approximately $5 \mathrm{mg} / \mathrm{ml}$ protein from mutant cells over-expressing FabG4 gave rise to an $\mathrm{NAD}(\mathrm{H})$-dependent oxidation of the $\mathrm{C}_{6}$ substrate at the rate of $0.21 \pm 0.02(\mathrm{SD}, \quad n=3) \mu \mathrm{mol} / \mathrm{mg}$ protein $\times \min ^{-1}$ (Table 2). When the $C_{10}$ substrate was used under the same reaction conditions, the soluble extracts containing FabG4 yielded a rate of $0.25 \pm 0.08(\mathrm{SD}, n=3) \mu \mathrm{mol} / \mathrm{mg}$ protein $\times$ $\min ^{-1}$ (Table 2). Further examination of FabG4 extracts with $\mathrm{NADP}^{+}$and $\mathrm{C}_{6}$ resulted in a rate of $3.5 \pm 0.3(\mathrm{SD}$, $n=3) \mathrm{nmol} / \mathrm{mg}$ protein $\times \min ^{-1}$ (Table 2), indicating that FabG4 might also utilize NADP(H), albeit inefficiently. Hence, heterologous expression of FabG4 in mutant oarl $\Delta$
Table 2 Enzyme activities in a yeast $\operatorname{oar} 1 \Delta$ mutant expressing FabG4

\begin{tabular}{lll}
\hline Substrate & \multicolumn{2}{l}{$\begin{array}{l}\text { 3-Oxoacyl-thioester reductase } \\
\text { activity }\left(\mathrm{nmol} / \mathrm{mg} \text { protein } \times \min ^{-1}\right)\end{array}$} \\
\cline { 2 - 3 } & $\mathrm{NAD}(\mathrm{H})$ & $\mathrm{NADP}(\mathrm{H})$ \\
\hline 2-trans-hexenoyl-CoA & $210 \pm 20^{\mathrm{a}}$ & $3.5 \pm 0.3$ \\
2-trans-decenoyl-CoA & $250 \pm 80$ & Not tested \\
\hline
\end{tabular}

a The values are means \pm standard deviations $(n=3)$ and represent averages of measurements using extracts obtained from three independent yeast cultures following overnight oleic acid induction. The yeast strain used was yPLM41

cells resulted in protein extracts containing readily detectable levels of 3-oxoacyl-thioester reductase activity.

FabG1 and FabG4 restore the assembly of cytochrome complexes in the oarl $1 \Delta$ mutant

Yeast cells carrying a deletion at the $O A R l$ gene have previously been shown to lack assembled cytochrome complexes (Schneider et al. 1997). Hence, rescue of oarl $\Delta$ cells from their fermentative phenotype should be associated with re-assembly of these structures. To verify this recovered function by means of a qualitative assay, the yeast strains described in "Yeast oarl $\Delta$ cells expressing FabG4 contain 3-oxoacyl-thioester reductase activity" were monitored for cytochrome complex assembly using the coldtemperature cuvette method (Lindenmayer and Estabrook 1958). Cell pastes were collected and applied to cuvettes that were then immersed in liquid nitrogen prior to being placed in a spectrophotometer. The result demonstrated that oarl $\Delta$ cells expressing peroxisomal Ctalp were not efficient at assembling the complete retinue of the cytochrome complexes (e.g., complexes $a$ and $a 3$; Fig. 3). On the other hand, the signals representing these complexes were more pronounced in mutant cells expressing native Oar1p or mycobacterial FabG1 and FabG4. Hence, this indicated that the ability of $\operatorname{oar} 1 \Delta$ mutants to grow on glycerol was coincidental with the recuperation of cytochrome complex assembly in their mitochondria.

Expression of FabG1 or FabG4 renews lipoic acid production in the oarl $\Delta$ mutant

Previous work demonstrated that the $\mathrm{C}_{8}$ octanoic acid product of mitochondrial FASII acts as the starting point for the synthesis of lipoic acid, and that a mutation in any one of the yeast genes encoding mitochondrial FASII enzyme causes a dramatic drop in the levels of lipoic acid (Hiltunen et al. 2005). However, this deficiency can be reversed when such mutants are supplied with known or presumed FASII enzyme activities of the corresponding type. To link FabG1 


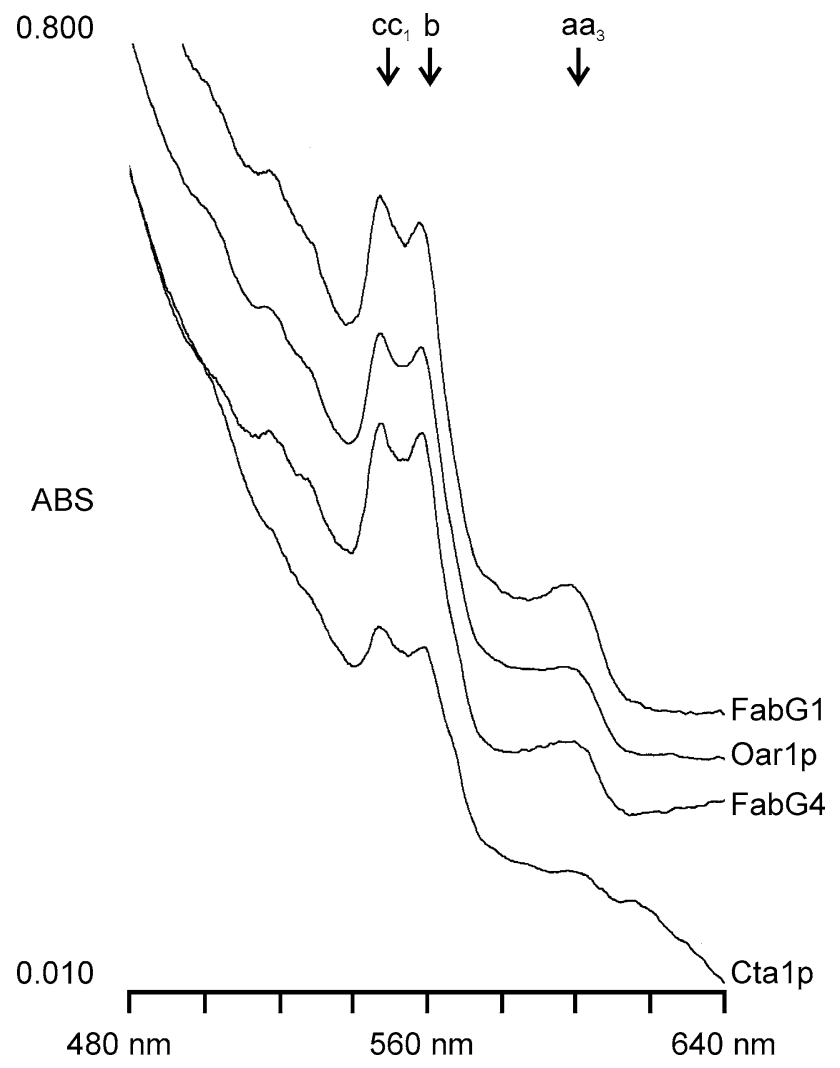

Fig. 3 Spectrophotometric assay for mitochondrial cytochrome complexes. Cell pastes from the indicated strains were collected from liquid cultures that were cultivated overnight in oleic acid medium and applied to the glass face of otherwise aluminum cold-temperature cuvettes that were immersed in liquid nitrogen prior to being placed in a spectrophotometer for analysis. The pastes were scanned at wavelengths between 480 and $640 \mathrm{~nm}$. Numbers to the left indicate units of absorbance (ABS), those at the bottom refer to wavelength in $\mathrm{nm}$. Peaks corresponding to known cytochromes are indicated (letters with arrows). The yPLM strains used were 37, 38, 41 and 43

and FabG4 expression with a rehabilitated FASII system in yeast mitochondria, lipoic acid levels were examined in the four strains using an in vivo growth assay that exploits a lipoic acid-deficient E. coli strain (Brody et al. 1997; Hayden et al. 1993). Extracts prepared from the negative control strain expressing Cta1p supported only a nominal growth level of these $E$. coli cells, commensurate with $7 \mathrm{ng}$ lipoic acid present per gram wet weight of yeast cells (Table 3). In contrast, yeast mutant cells expressing native Oarlp produced sufficient amounts of lipoic acid to support levels of bacterial growth equivalent to $193 \mathrm{ng}$ of lipoic acid per gram wet weight. Importantly, expression of FabG1 or FabG4 in the yeast mutant resulted in extracts containing the respective lipoic acid levels of 174 and $164 \mathrm{ng}$ of lipoic acid per gram wet weight, which were over 20 -fold higher than the levels of the negative control, thereby underscoring further the function of FabG4 as a putative FASII-like 3-oxoacyl-thioester reductase.
Table 3 Lipoic acid production in yeast oarl $\Delta$ mutants expressing the listed proteins

\begin{tabular}{ll}
\hline Protein & $\begin{array}{c}\text { Lipoic acid content } \\
\text { (ng/g [wet weight]) }\end{array}$ \\
\hline Cta1p & $7 \pm 2$ \\
Oar1p & $193 \pm 7^{\mathrm{a}}$ \\
FabG1 & $174 \pm 22$ \\
FabG4 & $164 \pm 23$ \\
\hline
\end{tabular}

a The values are means \pm standard deviations $(n=3)$ and represent averages of three independent bacterial growth responses. The $S$. cerevisiae yPLM strains used were 37, 38, 41 and 43

\section{Discussion}

The identification of a novel 3-oxoacyl-thioester reductase encoded by the essential mycobacterial gene fabG4 is described herein. The report takes into account the findings whereby FabG4 (1) restored the respiratory growth of an otherwise obligatory fermentative yeast mutant devoid of Oarlp; (2) contained high levels of 3-oxoacyl-thioester reductase activity; and (3) replaced Oarlp during lipoic acid synthesis. Future experimentation dedicated to knocking out $f a b G 4$ would likely help to elucidate whether FabG4 is in fact vital for M. tuberculosis persistence and virulence. Nevertheless, since FabG4 was practically indistinguishable from the essential mycobacterial 3-oxoacylAcpM reductase FabG1 within the parameters of phenotype rescue in yeast, it is attractive to speculate that FabG4 might also be involved in some hitherto elusive aspect of fatty acid biosynthesis in $M$. tuberculosis. Additional important results to emerge here regarding FabG1 include the refinement of its in vivo substrate specificities (down to $\mathrm{C}_{4}$ ), as well as exposure of its ability to function in yeast without resorting to protein-protein interactions with other mycobacterial FASII enzymes.

The role of FabG1 has been described previously within the context of mycobacterial FASII, which is outlined in great detail in Takayama et al. (2005). FASII begins with a $\mathrm{C}_{20}$ - CoA intermediate emerging from FASI, with additional rounds of $\mathrm{C}_{2}$ extensions being undertaken by FASII so as to arrive at the final length (up to $\mathrm{C}_{63}$ ) required for incorporation into mature mycolic acids. In a FASII prestep, 3-oxoacyl-AcpM synthase mtFabH (see below) converts the incoming $\mathrm{C}_{20}-\mathrm{CoA}$ to a $\left(\mathrm{C}_{22}\right)$ 3-oxoacyl-AcpM based on the availability of $\mathrm{C}_{3}$ malonyl-AcpM. Further FASII extensions are effected by two additional 3-oxoacylAcpM synthases, represented by KasA and KasB, giving rise to a 3-oxoacyl-AcpM intermediate. This metabolite acts as substrate for the aforementioned FabG1, which reduces the latter intermediary to 3-hydroxyacyl-AcpM, with the concomitant oxidation of NADPH. The subsequent 
two FASII steps include dehydration and reduction (Takayama et al. 2005).

With reference to the non-rescuing FabG homologs, the published data for FabG2, FabG3 and FabG5 remain rather scant. The crystal structure of FabG3/Rv2002 has been determined, but there are no data available on its physiological function or enzyme activity (Yang et al. 2002). Another homolog, FabG5/Rv2766c, was picked up with additional proteins (including InhA and the isoniazid-sensitive dihydrofolate reductase DfrA) in a screen for proteins capable of interacting with a resin mimicking the isoniazid-NAD/P adduct formed with the active species of isoniazid (Argyrou et al. 2006). Interestingly, isoniazid-sensitive FabG1 itself (Banerjee et al. 1998; Ducasse-Cabanot et al. 2004) was not identified in this search. In the aforementioned scan for genes needed for optimal growth (Sassetti et al. 2003), of the five $f a b G$ genes only $f a b G 2 / R v 1350$ was found to be required for this parameter. Moreover, none of them came up in a transposon-based screen for altered virulence (McAdam et al. 2002), or was upregulated in M. tuberculosis treated with isoniazid and other antimicrobials (Betts et al. 2003). Hence, further work will be required to underpin the functions of the remaining FabG homologs. This notwithstanding, it should be noted that since the present study was conducted entirely in a yeast background, the observations presented here may not necessarily apply to mycobacterial biochemistry. In other words, because phenotype rescue could not be achieved with the remaining three homologs, it would be premature to proclaim that they do not in fact play a role in fatty acid biosynthesis.

Returning to the essential requirement of FabG4 for mycobacterial existence, although an $M$. tuberculosis mutant knockout at $f a b G 4$ has been reported previously to be symptomless in the context of survival during infection (Sassetti and Rubin 2003), very important recent studies revealed $f a b G 4$ (as well as $f a b G 1, f a b D$ and $a c p S$, all affiliated with FASII) to be essential, at least in terms of growth of M. bovis BCG on Roisin's medium (Beste et al. 2009). Interestingly, the fabG4 locus ( $R v 0242 c)$ occurs in close proximity to that of $h t d X(R v 0241 c)$, whose latter product has been shown recently to act in yeast $h d t 2 \Delta$ cells as a physiological 3-hydroxyacyl-thioester dehydratase (Gurvitz et al. 2009). This represents the enzyme activity following FabG in the fatty acid biosynthesis scheme, and the two genes are predicted to occur in a single operon (operonDB; confidence $=84 ; n=57$ ). Since similar pair arrangements have been observed in M. tuberculosis with other genes of mycolic acid biosynthesis, including Rv1484/inhA and Rv1483/fabG1 (Takayama et al. 2005), it is at least plausible that HtdX and FabG4 might also function within a shared FASII-like pathway.

Regarding the issue of whether FabG1 is essential for pathogen survival, this has only recently been resolved.
A study dedicated to addressing this specific issue revealed that knocking out the gene for FabG1 in the pathogen was only possible if an additional plasmid-borne gene copy was made available, a procedure formally accepted as genetic proof for the essentiality of this gene (Parish et al. 2007). Although the requirement for FabG1 in M. tuberculosis could be compensated by using the homologous protein from M. smegmatis, functional complementation of the mycobacterial mutant devoid of its native FabG1 could not be achieved using an ectopically expressed $E$. coli FabG. It was argued that failure to complement the mutant phenotype using the bacterial protein was likely due to structural differences between the bacterial and mycobacterial proteins (Parish et al. 2007), in that the former is not able to accommodate long-chain fatty acids or undertake the suggested specific protein-protein interactions necessary for FASII (Veyron-Churlet et al. 2004, 2005).

Hence, the finding here that FabG1 could alleviate the growth phenotype of oarl $\Delta$ mutant cells was remarkable in light of the conclusions drawn regarding the central position of FabG1 in the complex presumed to exist within the structure of mycobacterial FASII (Veyron-Churlet et al. 2004). Using the yeast two-hybrid approach, native FabG1 was shown to interact only very weakly with the aforementioned $\mathrm{FabH}$, albeit subsequent co-immunoprecipitation failed to confirm this putative interaction altogether. Nevertheless, mutant versions of FabG1 were constructed so that they failed to form the native FabG1 multimer; these displayed more extensive protein-protein interactions, namely with the previously mentioned KasA, KasB, and FabH. Based on the results with these mutant FabG1 monomers, the authors concluded that the wild-type FabG1 monomer must also interact with each of the aforementioned condensing enzymes in the native state, and that the high propensity of these monomers to multimerize helps maintain the macromolecular organization of FASII. Since expression of these FabG1 mutants in M. tuberculosis appeared to block FASII, presumably by forming unproductive FabG1 multimers, this led to the suggestion that the quaternary structure of FabG1 is critical for a functional FASII complex (Veyron-Churlet et al. 2004). The conclusion that FabG1 represents a pivotal interacting protein that is vital for maintaining FASII complexes in M. tuberculosis is difficult to reconcile with the observations made here, whereby FabG1 could function in yeast FASII to $90 \%$ efficiency (Table 3) in an environment within which it has clearly not evolved to form highly specific protein-protein interactions.

A further point of contention regarding FabG1 is its physiological substrate specificity. Previous work on recombinant FabG1 showed that it was active in vitro using $\mathrm{C}_{4}$ acetoacetyl-CoA as substrate (Banerjee et al. 1998; Ducasse-Cabanot et al. 2004). However, other studies 
found that FabG1 preferentially acts on long-chain $\mathrm{C}_{8-20}$ CoA substrates, and that it metabolizes the $\mathrm{C}_{4}$ substrate only poorly (Marrakchi et al. 2002). Unfortunately, even following extensive over-expression in cells grown on oleic acid medium, the enzyme assay used here failed to detect any reductase activity for FabG1 using $\mathrm{C}_{6}$ or $\mathrm{C}_{10}$ substrates. Nevertheless, it can still be concluded that, at least within an in vivo yeast context, FabG1 was almost as efficient as native Oar1p in metabolizing short-chain $\mathrm{C}_{4-8}$ substrates, despite $f a b G 1$ being expressed at only modest levels commensurate with glucose derepression. Herein lays one of the major advantages of using yeast for examining the in vivo efficacy of enzymes for substrates that are otherwise not metabolized facilely in vitro. It is predicted that yeast FASII mutants will continue to serve as a convenient tool for identifying novel pathogen proteins.

Acknowledgments This work is dedicated to J. Kalervo Hiltunen, on the occasion of his 60th birthday. Johanna Mäkinen from the Mycobacterial Reference Laboratory at the National Public Health Institute in Turku, Finland, is thanked for providing M. tuberculosis H37Rv genomic DNA. J. Kalervo Hiltunen was instrumental for the enzyme assays and supported this work in a multitude of ways, Alexander J. Kastaniotis undertook the lipoic acid measurements and gave invaluable advice throughout this study, Zhi-Jun Chen is thanked for furnishing plasmid pYE352:OAR1, and Tatu Haataja for donating the hydratase 2 domain of D. melanogaster MFE2 (all from the University of Oulu, Finland). This work was funded by the grant P20764-B03 from the Austrian Science Fund (FWF).

Open Access This article is distributed under the terms of the Creative Commons Attribution Noncommercial License which permits any noncommercial use, distribution, and reproduction in any medium, provided the original author(s) and source are credited.

\section{References}

Argyrou A, Jin L, Siconilfi-Baez L et al (2006) Proteome-wide profiling of isoniazid targets in Mycobacterium tuberculosis. Biochemistry 45:13947-13953

Ausubel FM, Brent R, Kingston RE et al (1989) Current protocols in molecular biology. Wiley, NY

Banerjee A, Dubnau E, Quémard A et al (1994) inhA, a gene encoding a target for isoniazid and ethionamide in Mycobacterium tuberculosis. Science 263:227-230

Banerjee A, Sugantino M, Sacchettini JC et al (1998) The mabA gene from the inhA operon of Mycobacterium tuberculosis encodes a 3-ketoacyl reductase that fails to confer isoniazid resistance. Microbiology 144:2697-2704

Beste DJV, Espasa M, Bonde B et al (2009) The genetic requirements for fast and slow growth in mycobacteria. PLoS One 4:e5349

Betts JC, McLaren A, Lennon MG et al (2003) Signature gene expression profiles discriminate between isoniazid-, thiolactomycin-, and triclosan-treated Mycobacterium tuberculosis. Antimicrob Agents Chemother 47:2903-2913

Bloch K (1977) Control mechanisms for fatty acid synthesis in Mycobacterium smegmatis. Adv Enzymol 45:1-84

Bradford MM (1976) A rapid and sensitive method for the quantitation of microgram quantities of protein utilizing the principle of protein-dye binding. Anal Biochem 72:248-254
Brody S, Oh C, Hoja U et al (1997) Mitochondrial acyl carrier protein is involved in lipoic acid synthesis in Saccharomyces cerevisiae. FEBS Lett 408:217-220

Chen D-C, Yang B-C, Kuo T-T (1992) One-step transformation of yeast in stationary phase. Curr Genet 21:83-84

Chen Z-J, Kastaniotis AJ, Miinalainen IJ et al. (2009) 17 $\beta$-Hydroxysteroid dehydrogenase type 8 and carbonyl reductase type 4 assemble as a ketoacyl reductase of human mitochondrial FAS. FASEB J. doi:10.1096/fj.09-133587

Cole ST, Brosch R, Parkhill J et al (1998) Deciphering the biology of Mycobacterium tuberculosis from the complete genome sequence. Nature 393:537-544

Dommes V, Kunau W-H (1984) 2,4-Dienoyl coenzyme A reductases from bovine liver and Escherichia coli. Comparison of properties. J Biol Chem 259:1781-1788

Ducasse-Cabanot S, Cohen-Gonsaud M, Marrakchi H et al (2004) In vitro inhibition of the Mycobacterium tuberculosis $\beta$-ketoacylacyl carrier protein reductase MabA by isoniazid. Antimicrob Agents Chemother 48:242-249

Filipits M, Simon MM, Rapatz W et al (1993) A Saccharomyces cerevisiae upstream activating sequence mediates induction of peroxisome proliferation by fatty acids. Gene 132:49-55

Filppula SA, Sormunen RT, Hartig A et al (1995) Changing stereochemistry for a metabolic pathway in vivo. Experiments with the peroxisomal $\beta$-oxidation in yeast. J Biol Chem 270:27453-27457

Gerum AB, Ulmer JE, Jacobus DP et al (2002) Novel Saccharomyces cerevisiae screen identifies WR99210 analogues that inhibit Mycobacterium tuberculosis dihydrofolate reductase. Antimicrob Agents Chemother 46:3362-3369

Goldman P, Vagelos PR (1961) The specificity of triglyceride synthesis from diglycerides in chicken adipose tissue. J Biol Chem 236:2620-2623

Gurvitz A (2009) Caenorhabditis elegans F09E10.3 encodes a 3-oxoacyl-thioester reductase of mitochondrial type 2 fatty acid synthase FASII that is functional in yeast. J Biomed Biotechnol. http://www.hindawi.com/journals/jbb/aip.235868.html

Gurvitz A, Rottensteiner H, Kilpeläinen SH et al (1997) The Saccharomyces cerevisiae peroxisomal 2,4-dienoyl-CoA reductase is encoded by the oleate-inducible gene SPS19. J Biol Chem 272:22140-22147

Gurvitz A, Hiltunen JK, Kastaniotis AJ (2008a) Identification of a novel mycobacterial 3-hydroxyacyl-thioester dehydratase HtdZ (Rv0130) by functional complementation in yeast. J Bacteriol 190:4088-4090

Gurvitz A, Hiltunen JK, Kastaniotis AJ (2008b) Function of heterologous Mycobacterium tuberculosis InhA, a type 2 fatty acid synthase enzyme involved in extending $\mathrm{C}_{20}$ fatty acids to $\mathrm{C}_{60}$-to- $\mathrm{C}_{90}$ mycolic acids, during de novo lipoic acid synthesis in Saccharomyces cerevisiae. Appl Environ Microbiol 74:5078-5085

Gurvitz A, Hiltunen JK, Kastaniotis AJ (2009) Heterologous expression of mycobacterial proteins in yeast reveals two physiologically functional 3-hydroxyacyl-thioester dehydratases, HtdX and HtdY, in addition to HadABC and HtdZ. J Bacteriol 191:26832690

Hayden MA, Huang IY, Iliopoulos G et al (1993) Biosynthesis of lipoic acid: characterization of the lipoic acid auxotrophs Escherichia coli W1485-lip2 and JRG33-lip9. Biochemistry 32:37783782

Hill JE, Myers AM, Koerner TJ et al (1986) Yeast/E. coli shuttle vectors with multiple unique restriction sites. Yeast 2:163-167

Hiltunen JK, Okubo F, Kursu VA et al (2005) Mitochondrial fatty acid synthesis and maintenance of respiratory competent mitochondria in yeast. Biochem Soc Trans 33:1162-1165

Hsu AY, Poon WW, Shepherd JA et al (1996) Complementation of $\operatorname{coq} 3$ mutant yeast by mitochondrial targeting of the Escherichia coli UbiG polypeptide: evidence that UbiG catalyzes both 
$o$-methylation steps in ubiquinone biosynthesis. Biochemistry 35:9797-9806

Lindenmayer A, Estabrook RW (1958) Low-temperature spectral studies on the biosynthesis of cytochromes in baker's yeast. Arch Biochem Biophys 78:66-82

Marrakchi H, Ducasse S, Labess G et al (2002) MabA (FabG1), a Mycobacterium tuberculosis protein involved in the longchain fatty acid elongation system FAS-II. Microbiology 148:951-960

McAdam RA, Quan S, Smith DA et al (2002) Characterization of a Mycobacterium tuberculosis $\mathrm{H} 37 \mathrm{Rv}$ transposon library reveals insertions in 351 ORFs and mutants with altered virulence. Microbiology 148:2975-2986

Parish T, Roberts G, Laval F et al (2007) Functional complementation of the essential gene fabG1 of Mycobacterium tuberculosis by Mycobacterium smegmatis fabG but not Escherichia coli fabG. J Bacteriol 189:3721-3728

Rose MD, Winston F, Heiter P (1990) Methods in yeast genetics: a laboratory manual. Cold Spring Harbor Laboratory Press, Cold Spring Harbor

Rottensteiner H, Kal AJ, Filipits M et al (1996) Pip2p: a transcriptional regulator of peroxisome proliferation in the yeast Saccharomyces cerevisiae. EMBO J 15:2924-2934

Sambrook J, Fritsch EF, Maniatis T (1989) Molecular cloning: a laboratory manual. Cold Spring Harbor Laboratory, Cold Spring Harbor

Sassetti CM, Rubin EJ (2003) Genetic requirements for mycobacterial survival during infection. Proc Natl Acad Sci USA 100:1298912994
Sassetti CM, Boyd DH, Rubin EJ (2003) Genes required for mycobacterial growth defined by high density mutagenesis. Mol Microbiol 48:77-84

Schneider R, Brors B, Burger F et al (1997) Two genes of the putative mitochondrial fatty acid synthase in the genome of Saccharomyces cerevisiae. Curr Genet 32:384-388

Takayama K, Wang C, Besra GS (2005) Pathway to synthesis and processing of mycolic acids in Mycobacterium tuberculosis. Clin Microbiol Rev 18:81-101

Torkko JM, Koivuranta KT, Miinalainen IJ et al (2001) Candida tropicalis Etr1p and Saccharomyces cerevisiae Ybr026p (Mrf1'p), 2-enoyl thioester reductases essential for mitochondrial respiratory competence. Mol Cell Biol 21:6243-6253

Veyron-Churlet R, Guerrini O, Mourey L et al (2004) Protein-protein interactions within the fatty acid synthase-II system of Mycobacterium tuberculosis are essential for mycobacterial viability. Mol Microbiol 54:1161-1172

Veyron-Churlet R, Bigot S, Guerrini O et al (2005) The biosynthesis of mycolic acids in Mycobacterium tuberculosis relies on multiple specialized elongation complexes interconnected by specific protein-protein interactions. J Mol Biol 353:847-858

World Health Organisation (1997) Anti-tuberculosis drug resistance in the world: the WHO/IUATLD global project on anti-tuberculosis drug resistance surveillance. WHO Global Tuberculosis Programme, Geneva

Yang JK, Yoon HJ, Ahn HJ et al (2002) Crystallization and preliminary $\mathrm{X}$-ray crystallographic analysis of the Rv2002 gene product from Mycobacterium tuberculosis, a $\beta$-ketoacyl carrier protein reductase homologue. Acta Crystallogr D Biol Crystallogr 58:303-305 